\title{
Experimental $\mathcal{H}_{\infty}$ control to improve an industrial off-line tracking control scheme on an automotive suspension test rig
}

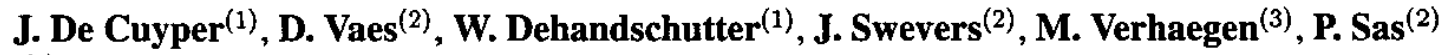 \\ (1) LMS International, Researchpark Haasrode Z1, Interleuvenlaan 68, 3001 Leuven, Belgium \\ (2) Katholieke Universiteit Leuven, Dept. of Mech. Eng., Celestijnenlaan 300B, 3001 Leuven, Belgium \\ (3) Techn. Univ. Delft, Control and Systems Eng. Laboratory, Mekelweg 4, 2528 CD, Delft, Netherlands \\ e-mail : joris.decuyper@lms.be
}

\begin{abstract}
This paper discusses the improvement of the tracking accuracy on an automotive suspension test rig by extending the available industrial off-line controller with a $\mathcal{H}_{\infty}$ feedback controller. To reach a prescribed accuracy level in a bandwidth between 0 and $20 \mathrm{~Hz}$, the off-line controller needs 7 iterations on a 2-axial durability suspension test rig to determine the correct control inputs. The extended scheme employs $\mathcal{H}_{\infty}$ control techniques to achieve the same result in only 3 iterations. This approach takes forward the process of durability testing to a point where the required accuracy can be achieved in significantly less time.
\end{abstract}

\section{Introduction}

In the automotive industry, one of the tests to sign-off a new vehicle prototype is a series of vibration tests on a hydraulic test rig. The idea is that, when the component or complete vehicle withstands the vibration test, it will not fail during its further lifetime. To make the vibration tests representative for the further life-time of the vehicle, reference forces (called targets) are measured during a test drive on a special test track and these target signals are reproduced on the test rig. The calculation of the input signals to be sent to the hydraulic actuators of the test rig such that the measured signals on the test rig match the target signals, is a multivariable tracking problem.

Current industry practice to solve this control problem is to use an off-line iterative control process $[5,2]$. This paper shows that, extending the current industrial off-line iterative control scheme with a $\mathcal{H}_{\infty}$ feedback controller, allows to reduce the required number of iterations significantly. Experimental evidence achieved on a suspension test rig is presented in support of the proposed concept.

The paper is organized as follows. Section 2 presents the currently used off-line control scheme as well as the extension with the $\mathcal{H}_{\infty}$ feedback controller. Section 3 describes the experimental set-up. Section 4 applies the industrial offline control scheme to reproduce the two target signals on the test rig. Section 5 describes the design of the vertical $\mathcal{H}_{\infty}$ feedback controller in detail. The design of the horizontal controller is completely analogue. Section $6 \mathrm{com}$ bines both SISO controllers and show that the combined control scheme (off-line $+\mathcal{H}_{\infty}$ ) allows to achieve the same accuracy as with the pure off-line scheme in less iterations. Section 7 summarizes the main conclusions of this paper.

\section{Control schemes}

2.1 The classically used industrial control scheme As already mentioned, controlling test rigs is a multivariable tracking problem. Dodds [4] was the first to suggest a frequency domain approach to solve this multivariable tracking problem. Today, this approach is widely accepted and used in industry for comfort and durability tests on vehicle prototypes [5]. Basically, it consists of two consecutive steps : an FRF identification and target simulation.

\section{FRF identification}

In this phase, a non-parametric frequency domain model (Frequency Response Function or FRF) is calculated. At each frequency $\omega$, the FRF matrix, denoted with $\hat{G}(j \omega)$ is estimated with the $H_{1}$-estimator [1] as

$$
[\hat{G}(j \omega)]=\left[\hat{S}_{y u}(j \omega)\right]\left[\hat{S}_{u t u}(j \omega)\right]^{-1}
$$

with $\left[\hat{S}_{y u}(j \omega)\right]$ the estimated input-output cross-spectrum at frequency $\omega$, and $\left[\hat{S}_{u u}(j \omega)\right]$ the estimated input autospectrum at frequency $\omega$.

Once the FRF model is calculated an inverse model $\hat{G}^{-1}(j \omega)$ is calculated by inverting the FRF matrix at each frequency $\omega$.

\section{Target simulation}

The target simulation is the control process itself, which calculates the correct control input signals.

The relation between the input vector $u(j \omega) \in \mathbb{C}^{p}$ and the output vector $y(j \omega) \in \mathbb{C}^{q}$ from the test rig is denoted with 
the function $G(u(j \omega)): \mathbb{C}^{p} \rightarrow \mathbb{C}^{q}$. The given reference data are denoted with $r(j \omega) \in \mathbb{C}^{q}$. In the remainder of the text, the dependency of the frequency $\omega$ is omitted to simplify the notation. The digital Fourier transform (DFT) is used to make the necessary transitions from time to frequency domain and vice versa.

To replicate the reference signal $r$, an iterative learning control (ILC, see for example [6]) process is used. An ILC scheme often uses little or no information from the system under control to calculate the control inputs. This is not the case here, because the previously identified FRF model $\hat{G}$, see equation (1), is explicitly used. Each iteration consists of the following 2 steps: the calculation of a new input signal, see equation (2) and an acquisition on the test rig, cfr. equation (3). The superscript $j$ with $j=1,2, \ldots$ refers to the iteration number and $Q^{j} \in \mathbb{R}^{q \times q}$ is a diagonal matrix, containing the iteration gains for iteration $j$. All elements $q^{j}$ of the matrix $Q^{j}$ satisfy $0 \leq q^{j} \leq 1$. Note also that $u^{0}$ and $y^{0}$ are zero vectors of appropriate size.

$$
\begin{aligned}
u^{j} & =u^{j-1}+\hat{G}^{-1} Q^{j}\left(r-y^{j-1}\right) \\
y^{j} & =G\left(u^{j}\right)
\end{aligned}
$$

Figure 1 gives a schematic representation of the equations (2)-(3) and represents the control scheme for the currently used off-line control process. The dashed lines in figure 1 stress the fact that the control scheme is an off-line process.

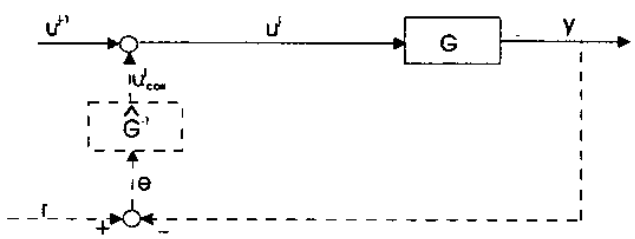

Figure 1: The classically used 'off-line control scheme'.

In the remainder of the text, the scheme of figure 1 is referred to as the 'off-line control scheme'. When a perfect model is available and hence $\hat{G}$ equals $G$, it is clear that the off-line control scheme will result in a decreasing tracking error and the replication of the reference signal $r$ can be achieved with arbitrary accuracy. The true value of the off-line scheme lies in its ability to deal with non-linear test rigs, in which case the plant model $\hat{G}$ is only a linear approximation of the non-linear plant $G$. The price to pay for this robustness is that it may require numerous iterations to achieve the required accuracy.

\subsection{The extended control scheme}

To reduce the number of required iterations, the control scheme of figure 2 is proposed. This scheme extends the control scheme of figure 1 with a real-time feedback loop.

In this scheme, $u_{f f}^{j}$ is calculated off-line in the same manner

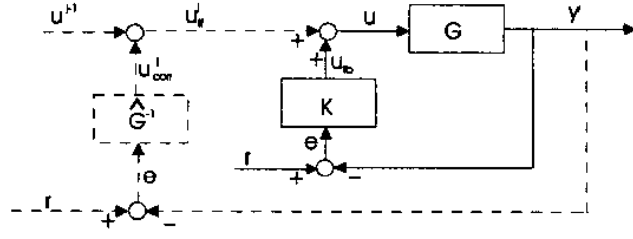

Figure 2: Extended control scheme: off-line feed-forward + realtime feedback

as with the off-line scheme (so by using the inverse of the open loop plant) and is used as a feed-forward signal. During the acquisition, the feedback controller $K$ calculates $u_{f b}$ which is added to $u_{f f}^{j}$ resulting in the total control input $u$. In the next iteration, this $u$ is used as the control input from the previous iteration to which $u_{\text {corr }}^{j}$ is added, resulting in the new feed-forward signal $u_{f f}^{j}$.

\subsection{Comparison of both control schemes}

[3] analyzes and compares both control schemes and shows that the tracking error in the $j$-th iteration is governed by following relations.

For the off-line control scheme,

$$
e^{j}=\left(I-(I+\Delta G) Q^{j}\right) e^{j-1}
$$

applies, where $e^{j}=r-y^{j}$ is the tracking error in the $j$ th iteration and $\Delta G$ denotes a multiplicative output uncertainty [9]. For the extended control scheme,

$$
e^{j}=S\left(I-(I+\Delta G) Q^{j}\right) e^{j-1},
$$

is valid. $S$ denotes the sensitivity function of the closed loop system.

Comparing equation (5) with (4) shows the advantage of using the real-time feedback controller. In each iteration, the tracking error is multiplied with an extra factor $S$. When $|S|$ is smaller than 1 , the feedback controller reduces the tracking error in each iteration and as such allows to reduce the number of iterations necessary to obtain a prescribed accuracy.

\section{Description of the test set-up}

The set-up is a multivariable ( 2 inputs, 2 outputs) suspension test rig which allows to excite the suspension in vertical and lateral direction, cfr. figure 3. At the top, see figure 3.a, the suspension is connected to a rigid frame; at the bottom the suspension is connected to the lower control arm, which in tum is connected via hinges to the same rigid frame. As figure 3.b shows, under the axle a connection with a hori- 
zontal rod and a vertically mounted fork is made. The actuators of the rig are connected to the rod and fork respectively and as such impose the motion of the rig.

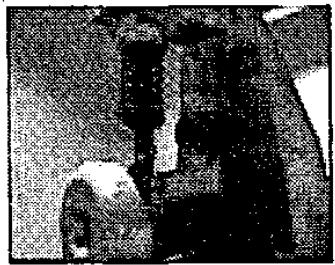

a.

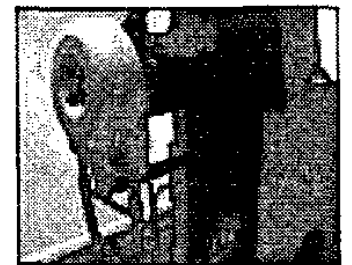

b.
Figure 3: Pictures of the test set-up. a: connection of top of suspension to rigid frame. $b$ : connection of the actuator rods to the suspension.

Separate digital PID controllers drive the actuators of the rig. The vertical actuator is displacement controlled, meaning that the vertical control input signal (input 1) is a displacement and the vertical PID controller tries to realize this displacement as close as possible by controlling the hydraulic servo valves. The horizontal actuator (input 2 ) is force controlled and the PID controller realizes a required input force by controlling the corresponding servo valves. When referring to figures 1 and 2, it is important to note that the PID controllers are part of the system $G$.

The 2 outputs of the system are the vertical and lateral forces, measured close to the actuators.

The targets which need to be reproduced are synthetic signals, because no forces measured during a test drive are available for that suspension. The control experiments consider the frequency band between 0 and $20 \mathrm{~Hz}$. All the experiments use a sample frequency of $1000 \mathrm{~Hz}$.

\section{Iterations with off-line control scheme}

This section discusses the results when applying the off-line control scheme, cfr. figure 1 and equations (2) and (3).

\subsection{FRF measurement}

A multisine [7] excitation is sent to both inputs. The resulting FRF, cfr. equation (1), is shown in figure 4.

When interpreting figure 4 , care needs to be taken because of the different units: the figure shows all values in $\mathrm{dB}$, but in principle, they have the unit of the respective output channel (both: $\mathrm{kN}$ ) divided by the unit of the respective input channel (input 1: $\mathrm{mm}$, input 2: $\mathrm{kN}$ ). This also means that a high amplitude of an element of the FRF matrix does not necessarily mean that a high coupling exists between the corresponding input and output, but can be due to the units in which the channels are expressed. The 'roughness' of the FRF is a better indicator to identify possible coupling effects. For example, when looking at the measured FRF matrix in figure 4, it is clear that the two diagonal elements are well determined. The amplitude and phase from the FRF between input 2 and output 1 are very noisy. So there is no coupling between the horizontal input force and the measured vertical force which is, from a physical point of view, an expected result. Secondly, the FRF between input 1 and output 2 shows that there exists some, but not very high coupling between the vertical input displacement and the horizontal force. The fact that the suspension does not move on a vertical straight line, but on a slightly inclined curve explains this observation.

\subsection{Non-linearities in the system}

The main source of non-linearity in the suspension is the damper. A simple sine test illustrates the non-linear behaviour. A sine with amplitude $10 \mathrm{~mm}$ and frequency 1 $\mathrm{Hz}$ excites the system. Figure 5 shows the resulting suspension force together with the vertical actuator displacement, which is directly proportional to the suspension compression.

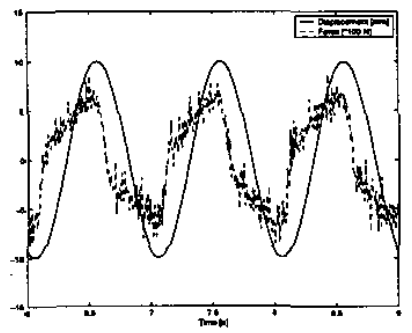

Figure 5: Illustration of non-linearity in the set-up. Vertical actuator displacement (solid) and resulting vertical force (dashed).

Figure 5 mainly shows two important effects. The first is that there is quite some noise on the measurement of the vertical force. Secondly, at each point in time when the displacement changes direction, i.e. when the suspension moves through an extreme position, the force drops suddenly, due to the presence of air in the damper chambers. The latter clearly is a non-linear effect.

\subsection{Target simulation}

The parameters to be determined during the target simulation are the iteration gains $Q^{j}$, cfr. equation (2). Following strategy has been followed: 4 iterations are performed with a gain of $30 \%$ for both channels, followed by 2 iterations with $50 \%$ gain. Then, a final 7 th iteration with $100 \%$ correction gain has been performed.

This strategy leads to the results shown in figure 6 . Figure 6 shows a comparison between the target (solid) and measured (dashed) response for the vertical (top) and horizontal (bottom) force. Both a time domain comparison (left) and frequency domain comparison (right) are shown.

An important metric to assess accuracy in a durability test 
Input 1:

Desired vert. displacement

Output 1:

Vert. force

Output 2:

Horiz. force
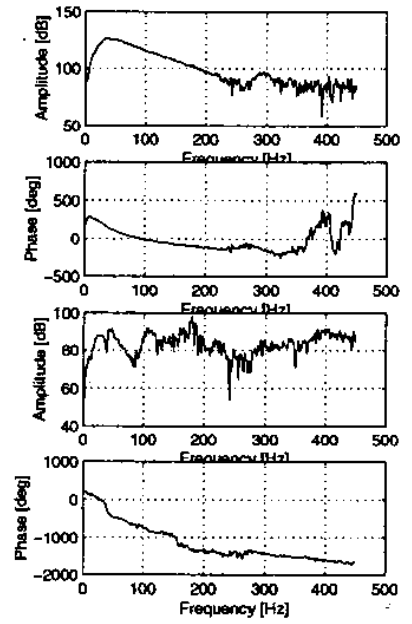

Input 2:

Desired horiz. force
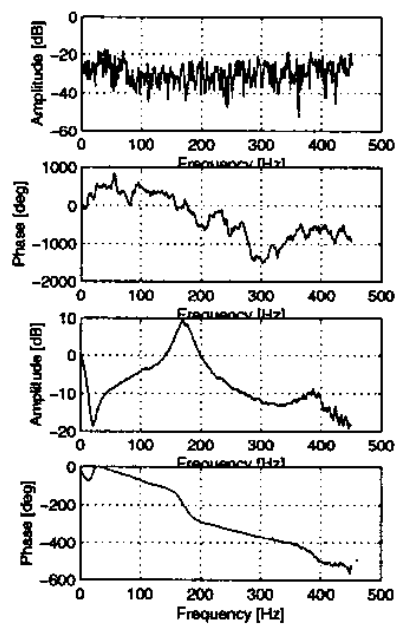

Figure 4: Measured FRF matrix. For each input-output pair, amplitude [dB] and phase [deg] are shown.

is the damage ratio, which is the ratio between the damage done to the suspension on the test rig on the one hand and the damage done to the suspension on the test track (during the measurement of the targets) on the other hand. In the ideal case, the damage ratio equals 1 (equal damage). The obtained accuracy here is satisfactory: after 7 iterations, the damage ratio between the target and measured response equals 0.92 for the vertical and 1.07 for the horizontal channel.

\section{Design of feedback controller for vertical channel}

This section describes the design of a $\mathcal{H}_{\infty}$ feedback controller for the vertical channel of the suspension test rig, that is for the SISO system between input 1 and output 1 .

\subsection{Identification of the state space model}

Frequency domain identification serves as a tool to identify a state space model. Main focus is to have a model which fits the measured FRF in a frequency band between 0 and $20 \mathrm{~Hz}$, but it is important that the fit outside this frequency band is not too bad either. In particular, there should be a good model fit in the cross-over region $[9,8]$. A strictly proper 8th order model appeared to be the best compromise between model complexity and model accuracy.

Figure 7.a shows that the state space models fits the FRF very accurately up to $200 \mathrm{~Hz}$. Important for a controller design are the poles and zeros of the identified model, cfr. figure 7.b. Note the presence of 2 RHP zeros (located at $1200 \pm 980 j$ ) which compromises the controller design significantly: their location corresponds to a frequency of $1540 \mathrm{rad} / \mathrm{s}$ or $245 \mathrm{~Hz}$. This means that the trade-off between $|S|$ smaller than 1 and $|S|$ larger than 1 needs to be
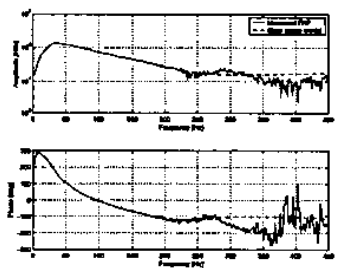

a.

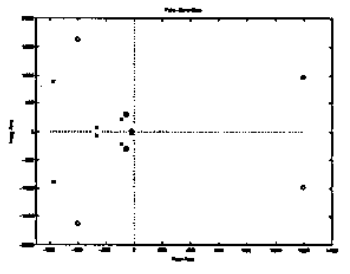

b.

Figure 7: The identified 8th order state space model. Figure a: comparison between the measured FRF (solid) and the model (dashed). Figure b: location of poles $(x)$ and zeros (o) in the complex plane.

made in the frequency band below $245 \mathrm{~Hz}$ [9].

\subsection{Controller design}

The controller is designed based on the mixed sensitivity approach [9], using 2nd order weighting functions on $S$ and T. This leads to the closed loop transfer functions shown in figure 8 .

Figure 8.a shows the amplitude of the sensitivity and complementary sensitivity function, respectively $S$ and $T$ with the inverse of their corresponding weighting functions $w_{P}$ and $w_{T}$. This figure shows that the closed loop bandwidth ${ }^{1}$ equals about $30 \mathrm{~Hz}$. The fact that $|S|$ exceeds 1 (and the corresponding loss of robustness and bandwidth limitation) is unavoidable due to the second waterbed formula (which applies due to the presence of the RHP zeros) [9].

Figure 8.b shows the Nyquist plot of the loopgain and allows to easily determine classical robustness indicators like low

${ }^{1}$ defined as the frequency where $|S|$ crosses $1 / \sqrt{2}=0.707$ from be- 

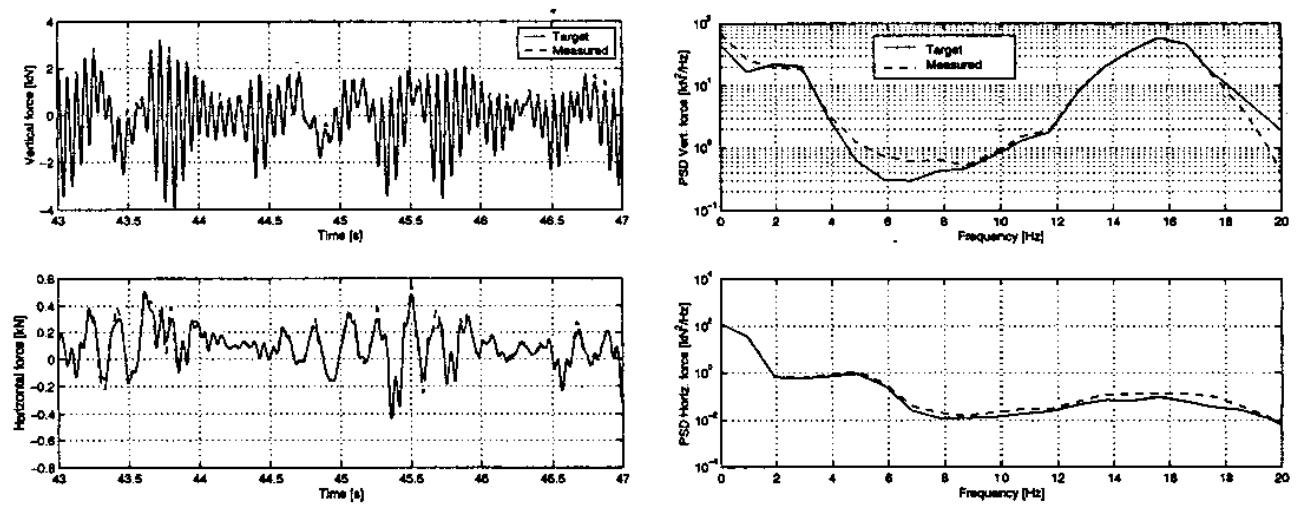

a.

b.

Figure 6: Illustration of obtained iteration quality with standard off-line process after 7 iterations. A time domain comparison (a) and frequency domain comparison (b) is shown between target (solid) and measurement (dashed). Obtained damage ratios are 0.92 (vertical) and 1.07 (horizontal)

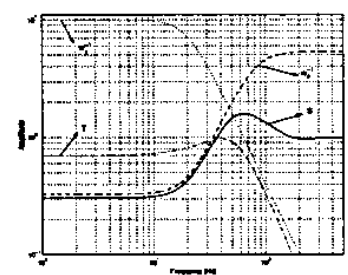

a.

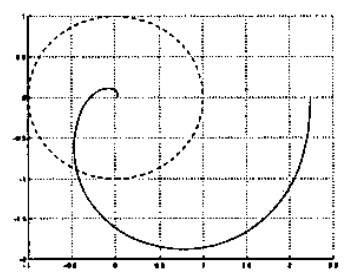

b.

Figure 8: Closed loop transfer functions for vertical axis. Figure a.: sensitivity function $S$ (solid) with weighting function $w_{P}$ (dashed) and complementary sensitivity function $\mathrm{T}$ (dashed-dotted) with weighting function $w_{T}$ (dotted). Figure b.: Nyquist plot of loopgain GK

phase and gain margin.

This concludes the design of the feedback controller for the vertical actuator. The design of the $\mathcal{H}_{\infty}$ controller for the horizontal axis (i.e. for the system between input 2 and output 2) is similar to that of the vertical controller and is therefore not discussed in detail here. However, the achievable performance and bandwidth without destabilizing the closed loop system is smaller than for the vertical controller.

\section{Iterations with real-time controller}

Combining the 2 SISO feedback controller into one diagonally structured MIMO controller and applying the combined control scheme (cfr. figure 2) leads after 3 iterations to the results shown in figure 9 . These results should be compared with the results shown in figure 6.

To show the evolution of the tracking accuracy, figure 10 shows $100 \% * \mid 1-$ damageratio $\mid$ as a function of the number of iterations.
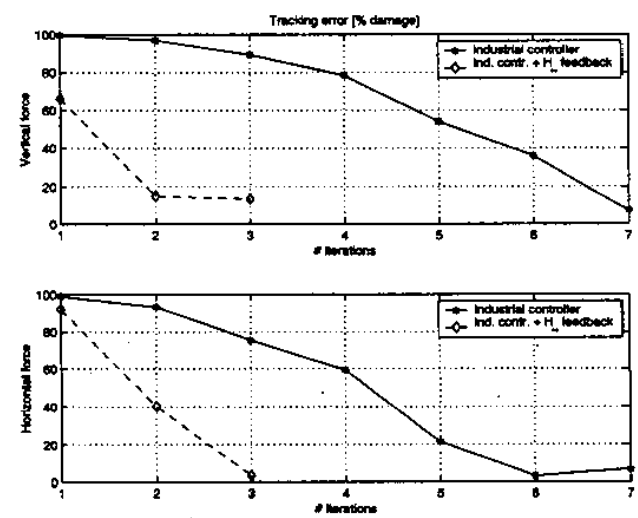

Figure 10: Comparison of accuracy between off-line control scheme (solid) with extended control scheme (dashed). Shown is $100 \% * \mid 1-$ damageratio $\mid$ versus the number of iterations

Figures 6,9 and figure 10 show that a comparable accuracy has been reached in less iterations ( 3 instead of 7 ) by extending the classically used off-line control scheme with a $\mathcal{H}_{\infty}$ feedback controller. In the industrial case, where the the target signals are long time sequences, the $\mathcal{H}_{\infty}$ controller can make a significant difference to the price tag of a durability test.

\section{Conclusions}

The conclusions of this paper can be summarized as follows.

- The experiments show that it is possible with the extended control scheme to control a non-linear plant and to reduce the number of required iterations to 

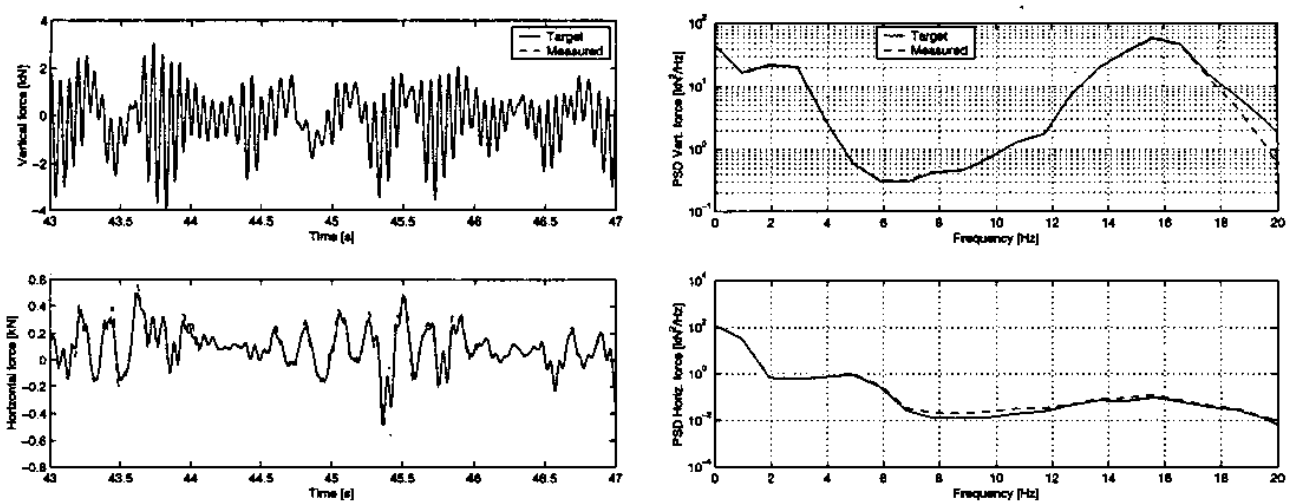

a.

b.

Figure 9: Illustration of obtained iteration quality with extended control scheme (after 3 iterations). A time domain comparison (a) and frequency domain comparison (b) is shown between target (solid) and measurement (dashed). Obtained damage ratios are 0.87 (vertical) and 1.01 (horizontal).

achieve a desired accuracy.

- A successful MIMO control experiment has been performed, where the MIMO controller consists of two SISO controllers.

\section{Acknowledgements}

This work was supported by the Flemish Institute for Innovation in Science and Technology (IWT) under contract number AUT-000104 (project name: 'FASTER') and by the Belgian programme on Interuniversity Poles of attraction initiated by the Belgian State, Prime Minister's Office, Science Policy Programming. The scientific responsibility remains with its authors.

\section{References}

[1] J.S. Bendat and A.G. Piersol. Engineering Applications of Correlation and Spectral Analysis. J.Wiley and Sons, New York, USA, 1980.

[2] J. De Cuyper, D. Coppens, C. Liefooghe, and J. Debille. Advanced System Identification Methods for improved Service Load Simulation on Multi Axial Test rigs - European Journal of Mechanical \& Environmental Engineering, 44(1):27-39, 1999. B.E.S.T Contributions (Belgian Environmental Sciences and Techniques Engineering).

[3] J. De Cuyper, M. Verhaegen, and J. Swevers. Offline feed-forward and $\mathcal{H}_{\infty}$ Feedback Control for improved Tracking on an industrial vibration test rig. Submitted to Control Engineering Practice, 2001. internal report nr 01 pp1 17 at KULeuven, Dept. of Mech. Engineering.

[4] C.J. Dodds. The Response of Vehicle Components to Random Road Surface Undulations. PhD thesis, Department of Mechanical Engineering, University of Glasgow, Scotland, 1972.
[5] C.J. Dodds and A.R. Plummer. Laboratory Road Simulation for Full Vehicle Testing: A Review. 7th Symposium on International Automotive Technology, SIAT, 10-13 Jan. 2001. 10-13 Jan. Pune, India. SAE paper No. 2001-010047.

[6] K. L. Moore. Iterative Learning Control: An Expository Overview, volume 1 of Applied and Computational Control, Signals, and Circuits, chapter 4, pages 151-214. Springer Verlag, New York, USA, 1999.

[7] R. Pintelon and J. Schoukens. System Identification: A Frequency Domain Approach. IEEE Press and J. Wiley and sons, Piscataway, NJ, USA, 2001.

[8] R.J.P. Schrama. Approximate Identification and Control Design. PhD thesis, Delft University of Technology, The Netherlands, 1992.

[9] S. Skogestad and I. Postlethwaite. Multivariable Feedback Control: Analysis and Design. J. Wiley and Sons, Chichester, UK, 1996. 